\title{
OPTIMAL STOPPING OF A SEQUENCE OF MAXIMA OVER AN UNOBSERVABLE SEQUENCE OF MAXIMA
}

0. Introduction. In this paper we consider some optimal stopping problems. Let $X_{1}, X_{2}, \ldots$ and $Y_{1}, Y_{2}, \ldots$ be independent, identically distributed real random variables defined on some fixed probability space $(\Omega, \mathscr{F}, P)$. We assume these two sequences are mutually independent, $X_{i}$ and $Y_{i}$ have the same continuous distribution function $F(x)$. Let

$$
\xi_{i}=\max \left(X_{1}, X_{2}, \ldots, X_{i}\right) \quad \text { and } \quad \eta_{i}=\max \left(Y_{1}, Y_{2}, \ldots, Y_{i}\right) \text {. }
$$

We observe only the sequence $X_{i}, i=1,2, \ldots$, and we want to stop it as soon as the value of $\xi_{i}$ is greater than $\eta_{i}$.

We can look at this problem as at the competition of two firms. Two competitive firms A and B are preparing a new product. The design offices of both firms produce new offers in each time unit. The attractiveness of the propositions is estimated in each firm separately. As the actual offers in each firm these ones are accepted whose estimates are the highest up to the moment. The firm A has priority in coming into the market. A must do it at such a moment when $B$ does not dispose of a better offer than $A$.

Now we precise the problem under consideration. Let us put $\mathscr{F}_{i}=\sigma\left\{X_{1}, X_{2}, \ldots, X_{i}\right\}$, let $\mathfrak{M}$ denote the set of all Markov moments $\tau$ with respect to $\left\{\mathscr{F}_{i}\right\}_{i=1}^{\infty}$ and $\mathfrak{M}^{N}=\{\tau \in \mathfrak{M}: \tau \leqslant N\}$. We consider the following cases:

(a) We search for $\tau^{*} \in \mathfrak{M}^{N}$ such that

$$
P\left\{\xi_{\tau^{*}}>\eta_{\tau^{*}}\right\}=\sup _{\tau \in \mathfrak{M}^{N}} P\left\{\xi_{\tau}>\eta_{\tau}\right\}
$$

(b) We must determine $\tau^{*} \in \mathfrak{M}$ such that $P\left\{\tau^{*}<\infty\right\}=1$ and

where $A$ is some real constant.

$$
P\left\{A \geqslant \xi_{\tau^{*}}>\eta_{\tau^{*}}\right\}=\sup _{\tau \in \mathfrak{M}} P\left\{A \geqslant \xi_{\tau}>\eta_{\tau}\right\}
$$

(c) We search for $\tau^{*} \in \mathfrak{M}$ such that $P\left\{\tau^{*}<\infty\right\}=1$ and

where $A$ is some real constant.

$$
P\left\{\min \left(A, \xi_{\tau^{*}}\right)>\eta_{\tau^{*}}\right\}=\sup _{\tau \in \mathfrak{M}} P\left\{\min \left(A, \xi_{\tau}\right)>\eta_{\tau}\right\},
$$


(d) We are to determine $\tau^{*} \in \mathfrak{M}$ such that $P\left\{\tau^{*}<\infty\right\}=1$ and

$$
\mathrm{E} \alpha^{\tau^{*}} \chi_{\left\{\xi_{\tau^{*}}>\eta_{\left.\tau^{*}\right\}}\right.}=\sup _{\tau \in \mathfrak{M}} \mathrm{E} \alpha^{\tau} \chi_{\left\{\xi_{\tau}>\eta_{\tau}\right\}}
$$

where $\alpha \in(0,1)$ and $\chi_{A}$ is the characteristic function of the set $A$.

The problem of the optimal stopping of the two sequences of maxima when only one sequence is observed was not investigated in the literature. The problem of stopping at the moment when the maximum of the sequence is attained has been investigated in [1] and [2].

Our problem can be reduced to the optimal stopping for the Markov chain [3]. We present the way of this reduction for case (a). Other problems can be treated similarly.

Let $Z_{n}=P\left\{\xi_{n}>\eta_{n} \mid \mathscr{F}_{n}\right\}, n=1,2, \ldots$ Then we obtain

$$
P\left\{\xi_{\tau}>\eta_{\tau}\right\}=\mathrm{E} Z_{\tau}
$$

for $\tau \in \mathfrak{M}$ such that $P\{\tau<\infty\}=1$.

Since $F$ is continuous, $F\left(X_{i}\right)$ is uniformly distributed in $[0,1]$, and

$$
P\left\{X_{i}>Y_{i} \Leftrightarrow F\left(X_{i}\right)>F\left(Y_{i}\right)\right\}=1,
$$

without loss of generality we may additionally assume that $\boldsymbol{X}_{i}$ and $\boldsymbol{Y}_{\boldsymbol{i}}$, $i=1,2, \ldots$, are uniformly distributed themselves.

By the last assumption we have $Z_{n}=\xi_{n}^{n}=g\left(n, \xi_{n}\right)$. Now, $\xi=\left(\xi_{n}\right)_{n=0}^{\infty}$ is the Markov chain with respect to $\left\{\mathscr{F}_{n}\right\}_{n=0}^{\infty}$ with the state space $[0,1]$ and the transition probability function

$$
p(n, x ; n+1, B)= \begin{cases}x+|B \cap(x, 1]| & \text { if } x \in B \\ |B \cap(x, 1]| & \text { if } x \notin B\end{cases}
$$

for $n=0,1, \ldots, x \in[0,1]$, where $B$ is a Borel subset of $[0,1]$, and $|\cdot|$ stands for the Lebesgue measure.

Thus we transform the initial problem (in case (a)) into the optimal stopping of the Markov chain $\xi$ with the reward function $g(n, x)=x^{n}$ for $x \in[0,1], n=1,2, \ldots, N$.

The optimal stopping problem for the finite Markov chain $\xi=\left(\xi_{n}\right)_{n=0}^{N}$ with reward function $g(n, x)$ has the following solution:

Let $\xi_{n}=x, \mathfrak{M}_{n}^{N}=\left\{\tau \in \mathfrak{M}^{N}: \tau \geqslant n\right\}$, and

$$
v_{N}(n, x)=\sup _{\tau \in \mathfrak{M}_{n}^{N}} \mathrm{E}_{x} g\left(\tau, \xi_{\tau}\right)
$$

write also

and

$$
T g(n, x)=\mathrm{E}_{x} g\left(n+1, \xi_{1}\right)
$$

$$
Q g(n, x)=\max \{g(n, x), T g(n, x)\}
$$


LEMMA 1 ([3], p. 106-108). Let $\xi=\left(\xi_{n}\right)_{n=0}^{N}$ be a homogeneous Markov chain with a state space $E$ and let $g:\{0,1, \ldots, N\} \times E \rightarrow R$ be a bounded nonnegative function. Then the function $v_{N}(n, x)$ satisfies the equation

and the moment

$$
v_{N}(n, x)=\max \left\{g(n, x), T v_{N}(n, x)\right\}=Q^{N-n} g(n, x),
$$

$$
\tau_{n}^{* N}=\min \left\{n \leqslant k \leqslant N: v_{N}\left(k, \xi_{k}\right)=g\left(k, \xi_{k}\right)\right\}
$$

is optimal in $\mathfrak{M}_{n}^{N}$. The optimal gain is $v_{N}(x)=v_{N}(0, x)$ and the optimal stopping time is $\tau_{0}^{* N}$.

In cases (b), (c), and (d) the reduction leads to the optimal stopping problem for the infinite Markov chain with some bounded reward functions. The optimal stopping problem for the infinite Markov chain with reward function $g(n, x)$ has the following solution:

Let $\xi_{n}=x, \mathfrak{M}_{n}=\{\tau \in \mathfrak{M}: \tau \geqslant n\}$, and

$$
v(n, x)=\sup _{\tau \in \mathbb{M}_{n}} \mathrm{E}_{x} g\left(\tau, \xi_{\tau}\right)
$$

LEMMA 2 ([3], p. 106-108). Let $\xi=\left(\xi_{n}\right)_{n=0}^{\infty}$ be a homogeneous Markov chain with a state space $E$ and let $g:\{0,1, \ldots\} \times E \rightarrow R$ be a bounded nonnegative function. Then the function $v(n, x)$ satisfies the equations

and

$$
v(n, x)=\max \{g(n, x), T v(n, x)\}
$$

The moment

$$
v(n, x)=\lim _{N \rightarrow \infty} Q^{N} g(n, x)
$$

$$
\tau_{n, \varepsilon}^{*}=\inf \left\{m \geqslant n: v\left(m, \xi_{m}\right) \leqslant g\left(m, \xi_{m}\right)+\varepsilon\right\}, \quad \varepsilon>0,
$$

is e-optimal in $\mathfrak{M}_{n}$, i.e.,

$$
v(n, x) \leqslant \mathrm{E}_{x} g\left(\tau_{n, \varepsilon}^{*}, \xi_{\tau_{n, 8}^{*}}\right)+\varepsilon .
$$

If $P\left\{\tau_{0,0}^{*}<\infty\right\}=1$, then $\tau_{0,0}^{*}$ is the optimal stopping time and $v(x)=v(0, x)$.

1. The optimal stopping of the finite sequence of maxima. The remarks about the reduction of case (a) to the optimal stopping problem for the Markov chain $\xi=\left(\xi_{n}\right)_{n=0}^{N}$ with the transition probability function (1) and the reward function $g(n, x)=x^{n}$ for $x \in[0,1], n=1,2, \ldots, N$, and Lemma 1 give us the following

THEOREM 1. There exists a sequence of numbers $x_{1} \leqslant x_{2} \leqslant \ldots \leqslant x_{N-1}, x_{N}$ $=0$ such that $\tau^{*}=\min \left\{1 \leqslant n \leqslant N: \xi_{n} \geqslant x_{n}\right\}$ satisfies the condition

$$
\begin{aligned}
P\left\{\xi_{\tau^{*}}>\eta_{\tau^{*}}\right\} & =\sup _{\tau \in \mathfrak{R}^{N}} P\left\{\xi_{\tau}>\eta_{\tau}\right\} \\
& =\frac{1}{2}\left(\frac{1}{2 N-1} x_{1}^{2 N}-x_{1}^{2}+1\right)+\psi_{1}\left(x_{2}\right) x_{1},
\end{aligned}
$$


where $\psi_{1}$ can be derived from the recursive equalities

$$
\begin{gathered}
\psi_{N-1}\left(x_{N}\right)=\frac{1}{N+1}, \\
\psi_{N-i}\left(x_{N-i+1}\right)=\frac{N}{(N+i)(N+i-1)} x_{N-i+1}^{N+i}-\frac{1}{N-i+2} x_{N-i+1}^{N-i+2}+ \\
+\psi_{N-i+1}\left(x_{N-i+2}\right) x_{N-i+1}+\frac{1}{N-i+2}, \quad i=2,3, \ldots, N-1,
\end{gathered}
$$

and $x_{N-i}$ for $i=1,2, \ldots, N-1$ is the unique solution of the equation

$$
\frac{N}{N+i} x^{N+i}+\psi_{N-i}\left(x_{N-i+1}\right)-x^{N-i}=0
$$

in the interval $(0,1)$.

For the proof we show first the following lemma:

LEMMA 3. The equations

$$
\mathrm{E}_{x} g\left(n+1, \xi_{1}\right)=g(n, x)
$$

have in $(0,1)$ the unique solution $x_{n}^{0}$ for $n=1,2, \ldots, N-1$ such that $x_{n}^{0} \leqslant x_{n+1}^{0}$ and $\mathrm{E}_{x} g\left(n+1, \xi_{1}\right) \leqslant g(n, x)$ for $x \geqslant x_{n}^{0}$.

Proof. Let us put

$$
h(n, x)=\mathrm{E}_{x} g\left(n+1, \xi_{1}\right)-g(n, x) .
$$

The functions $h(n, x), n=1,2, \ldots, N-1$, are continuous and have the following properties:

(i) $h(n, 0)=1 /(n+2), h(n, 1)=0, n=1,2, \ldots, N-1$;

(ii) $h(n, x)$ has a unique minimum at $\alpha_{1}^{n}=\sqrt{n /(n+1)}$.

Therefore, equations (2) have unique solutions $x_{n}^{0}$ and $x_{n}^{0}<a_{1}^{n}$, $n=1,2, \ldots, N-1$.

Let us write

$$
\varphi_{n}(x)=\frac{(n+2) h(n, x)}{1-x}
$$

We have

$$
\varphi_{n+1}(x)-\varphi_{n}(x)=-x^{n}\left[(n+2) x^{2}+x-(n+2)\right] .
$$

The equation $\varphi_{n+1}(x)-\varphi_{n}(x)=0$ has in $(0,1)$ the unique solution $\alpha_{2}^{n}$. We can check that $\alpha_{1}^{n}<\alpha_{2}^{n}$. Consequently, $\varphi_{n+1}(x)-\varphi_{n}(x)>0$ for $x \in\left(0, \alpha_{2}^{n}\right)$, $n=1,2, \ldots, N-1$, and $h\left(n+1, \alpha_{2}^{n}\right)<0$. Therefore, $x_{n+1}^{0} \geqslant x_{n}^{0}$, which completes the proof of Lemma 3 . 
Proof of Theorem 1. By Lemmas 1 and 3 we have

$$
\begin{aligned}
v_{N}(N-1, x) & =\max \left\{g(N-1, x), \mathrm{E}_{x} g\left(N-1, \xi_{1}\right)\right\} \\
& = \begin{cases}x^{N-1} & \text { for } x \geqslant x_{N-1} \\
\frac{N}{N+1} x^{N+1}+\frac{1}{N+1} & \text { for } x<x_{N-1}\end{cases}
\end{aligned}
$$

where $x_{N-1}=x_{N-1}^{0}$ is defined in Lemma 3. By definition, $x_{N}=0$.

Let us put

(3) $f(N-j, x)=\mathrm{E}_{x} v_{N}\left(N-j+1, \xi_{1}\right)-x^{N-j}, \quad j=2,3, \ldots, N-1$.

We assume by induction that we have obtained $x_{N-1}>x_{N-2}>\ldots>x_{N-i+1}$ for $i<N-1$ and $x_{N-i+1}>x_{N-i+1}^{0}$. Moreover,

$$
v_{N}(N-j, x)= \begin{cases}x^{N-j} & \text { for } x \geqslant x_{N-j} \\ \frac{N}{N+j} x^{N+j}+\psi_{N-j}\left(x_{N-j+1}\right) & \text { for } x<x_{N-j}\end{cases}
$$

for $j=1,2, \ldots, i-1$, where

$$
\begin{gathered}
\psi_{N-1}\left(x_{N}\right)=\frac{1}{N+1}, \\
\psi_{N-j}\left(x_{N-j+1}\right)=\frac{N}{(N+j)(N+j-1)} x_{N-j+1}^{N+j}-\frac{1}{N-j+2} x_{N-j+1}^{N-j+2}+ \\
+\psi_{N-j+1}\left(x_{N-j+2}\right) x_{N-j+1}+\frac{1}{N-j+2}, \quad j=2,3, \ldots, N-1,
\end{gathered}
$$

and $x_{N-j}$ is the unique solution of the equation $f(N-j, x)=0$ in $\left(0, x_{N-j+1}\right)$. We determine $x_{N-i}$ and $v_{N}(N-i, x)$.

If $x<x_{N-i+1}$, then

$$
\begin{aligned}
\mathrm{E}_{x} v_{N}\left(N-i+1, \xi_{1}\right)= & \frac{N}{N+i-1} x^{N+i-1} x+\psi_{N-i+1}\left(x_{N-i+2}\right) x+ \\
& +\int_{x}^{x_{N-i+1}}\left[\frac{N}{N+i-1} y^{N+i-1}+\psi_{N-i+1}\left(x_{N-i+2}\right)\right] d y+ \\
& +\int_{x_{N-i+1}}^{1} y^{N-i+1} d y=\frac{N}{N+i} x^{N+i}+\psi_{N-i}\left(x_{N-i+1}\right)
\end{aligned}
$$


If $x \geqslant x_{N-i+1}$, then

$$
\begin{aligned}
\mathrm{E}_{x} v_{N}\left(N-i+1, \xi_{1}\right) & =x^{N-i+2} x+\int_{x}^{1} y^{N-i+1} d y \\
& =\frac{N-i+1}{N-i+2} x^{N-i+2}+\frac{1}{N-i+2}
\end{aligned}
$$

The function $f(N-i, x)$ defined by $(3)$ is continuous in $(0,1)$ and has the following properties:

(i) For $x \geqslant x_{N-i+1}, f(N-i, x)<0$ as $h(N-i+1, x)=0$ has the $\operatorname{root} x_{N-i+1}^{0}, x_{N-i+1}^{0}<x_{N-i+1} ; h(N-i, x)<0$ for $x>x_{N-i}^{0}, x_{N-1}^{0}<x_{N-i+1}^{0}$ $<x_{N-i+1}$ and $f(N-i, x)=h(N-i, x)<0$ for $x \geqslant x_{N-i+1}$.

(ii) For $x<x_{N-i+1}, f(N-i, x)>h(N-i, x)$ since $v_{N}(N-i+1, x)$ $>g(N-i+1, x)$ for $x<x_{N-i+1}$.

(iii) $f(N-i, x)$ is a decreasing function for $x<x_{N-i+1}$.

These facts imply that there exists a unique solution of the equation $h(N-i, x)=0$ in $(0,1), x_{N-i}^{0}<x_{N-i}<x_{N-i+1}$, and $h(N-i, x)>0$ in $\left[0, x_{N-i}\right)$. Thus $v_{N}(N-i, x)$ is given by (4). Assuming that $P\left\{\xi_{0}=0\right\}=1$ we obtain

$$
v(0,0)=\mathrm{E}_{0} v_{N}\left(1, \xi_{1}\right)=\frac{1}{2}\left(\frac{1}{2 N-1} x_{1}^{2 N}-x_{1}^{2}+1\right)+\psi_{1}\left(x_{2}\right) x_{1} .
$$

This completes the proof of Theorem 1 .

Table 1 gives $x_{1}, x_{2}, \ldots, x_{N-1}$ and $v_{N}(0,0)$ for some values of $N$. The values $x_{1}, x_{2}, \ldots, x_{N-1}$ are written row-wise (with 7 entries in each row).

2. The optimal stopping of the infinite sequence of maxima. In this section we consider cases (b) and (c). Since we assume that $X_{i}$ and $\boldsymbol{Y}_{i}$ have the uniform distribution on $[0,1]$, we have $A \in(0,1)$.

We search for $\tau^{*} \in \mathfrak{M}$ such that

$$
P\left\{A \geqslant \xi_{\tau^{*}}>\eta_{\tau^{*}}\right\}=\sup _{\tau \in \mathfrak{N}} P\left\{A \geqslant \xi_{\tau}>\eta_{\tau}\right\}
$$

Let us put

$$
Z_{n}=P\left\{A \geqslant \xi_{n}>\eta_{n} \mid F_{n}\right\}=g\left(n, \xi_{n}\right)= \begin{cases}0 & \text { for } \xi_{n}>A \\ \xi_{n}^{n} & \text { for } \xi_{n} \leqslant A\end{cases}
$$

$\xi=\left(\xi_{n}\right)_{n=0}^{\infty}$ is the Markov chain with respect to $\left\{\mathscr{F}_{n}\right\}_{n=0}^{\infty}$ with the state space $[0,1]$ and the transition probability function (1). 
TABLE I

\begin{tabular}{|c|c|c|c|c|c|c|c|c|}
\hline$N$ & \multicolumn{7}{|c|}{$x_{1}, x_{2}, \ldots, x_{N-1}$} & $v$ \\
\hline 1 & & & & 2 & & & & 3 \\
\hline 2 & 0.3660 & & & & & & & 0.5580 \\
\hline 3 & 0.4322 & 0.5774 & & & & & & 0.5903 \\
\hline 4 & 0.4747 & 0.6190 & 0.6836 & & & & & 0.6114 \\
\hline 5 & 0.5048 & 0.6480 & 0.7106 & 0.7472 & & & & 0.6269 \\
\hline 6 & 0.5274 & 0.6697 & 0.7305 & 0.7659 & 0.7895 & & & 0.6389 \\
\hline 7 & 0.5453 & 0.6866 & 0.7460 & 0.7803 & 0.8032 & 0.8197 & & 0.6485 \\
\hline 8 & 0.5597 & 0.7002 & 0.7585 & 0.7919 & 0.8141 & 0.8301 & 0.8423 & 0.6566 \\
\hline 9 & $\begin{array}{l}0.5718 \\
0.8599\end{array}$ & 0.7114 & 0.7689 & 0.8015 & 0.8231 & 0.8386 & 0.8505 & 0.6634 \\
\hline 20 & $\begin{array}{l}0.5821 \\
0.8665\end{array}$ & $\begin{array}{l}0.7209 \\
0.8740\end{array}$ & 0.7776 & 0.8096 & 0.8306 & 0.8458 & 0.8573 & 0.6694 \\
\hline 11 & $\begin{array}{l}0.5910 \\
0.8721\end{array}$ & $\begin{array}{l}0.7291 \\
0.8794\end{array}$ & $\begin{array}{l}0.7851 \\
0.8854\end{array}$ & 0.8165 & 0.8371 & 0.8519 & 0.8632 & 0.6746 \\
\hline 12 & $\begin{array}{l}0.5988 \\
0.8770\end{array}$ & $\begin{array}{l}0.7361 \\
0.8840\end{array}$ & $\begin{array}{l}0.7915 \\
0.8900\end{array}$ & $\begin{array}{l}0.8224 \\
0.8950\end{array}$ & 0.8427 & 0.8572 & 0.8683 & 0.6793 \\
\hline 13 & $\begin{array}{l}0.6057 \\
0.8812\end{array}$ & $\begin{array}{l}0.7423 \\
0.8881\end{array}$ & $\begin{array}{l}0.7972 \\
0.8940\end{array}$ & $\begin{array}{l}0.8278 \\
0.8989\end{array}$ & $\begin{array}{l}0.8477 \\
0.9031\end{array}$ & 0.8619 & 0.8727 & 0.6834 \\
\hline 14 & $\begin{array}{l}0.6120 \\
0.8850\end{array}$ & $\begin{array}{l}0.7478 \\
0.8918\end{array}$ & $\begin{array}{l}0.8023 \\
0.8974\end{array}$ & $\begin{array}{l}0.8325 \\
0.9023\end{array}$ & $\begin{array}{l}0.8520 \\
0.9064\end{array}$ & $\begin{array}{l}0.8661 \\
0.9100\end{array}$ & 0.8766 & 0.6872 \\
\hline 15 & $\begin{array}{l}0.6175 \\
0.8884\end{array}$ & $\begin{array}{l}0.7527 \\
0.8950\end{array}$ & $\begin{array}{l}0.8068 \\
0.9006\end{array}$ & $\begin{array}{l}0.8366 \\
0.9053\end{array}$ & $\begin{array}{l}0.8560 \\
0.9094\end{array}$ & $\begin{array}{l}0.8696 \\
0.9129\end{array}$ & $\begin{array}{l}0.8802 \\
0.9161\end{array}$ & 0.6907 \\
\hline 16 & $\begin{array}{l}0.6226 \\
0.8914 \\
0.9213\end{array}$ & $\begin{array}{l}0.7571 \\
0.8980\end{array}$ & $\begin{array}{l}0.8108 \\
0.9034\end{array}$ & $\begin{array}{l}0.8404 \\
0.9080\end{array}$ & $\begin{array}{l}0.8595 \\
0.9120\end{array}$ & $\begin{array}{l}0.8731 \\
0.9155\end{array}$ & $\begin{array}{l}0.8833 \\
0.9186\end{array}$ & 0.6938 \\
\hline 27 & $\begin{array}{l}0.6273 \\
0.8942 \\
0.9236\end{array}$ & $\begin{array}{l}0.7612 \\
0.9006 \\
0.9260\end{array}$ & $\begin{array}{l}0.8145 \\
0.9060\end{array}$ & $\begin{array}{l}0.8438 \\
0.9105\end{array}$ & $\begin{array}{l}0.8627 \\
0.9144\end{array}$ & $\begin{array}{l}0.8761 \\
0.9179\end{array}$ & $\begin{array}{l}0.8862 \\
0.9209\end{array}$ & 0.6968 \\
\hline 18 & $\begin{array}{l}0.6316 \\
0.8967 \\
0.9256\end{array}$ & $\begin{array}{l}0.7649 \\
0.9030 \\
0.9280\end{array}$ & $\begin{array}{l}0.8178 \\
0.9083 \\
0.9301\end{array}$ & $\begin{array}{l}0.8469 \\
0.9128\end{array}$ & $\begin{array}{l}0.8656 \\
0.9166\end{array}$ & $\begin{array}{l}0.8789 \\
0.9200\end{array}$ & $\begin{array}{l}0.8888 \\
0.9230\end{array}$ & 0.6995 \\
\hline 19 & $\begin{array}{l}0.6356 \\
0.8990 \\
0.9274\end{array}$ & $\begin{array}{l}0.7682 \\
0.9052 \\
0.9298\end{array}$ & $\begin{array}{l}0.8209 \\
0.9104 \\
0.9319\end{array}$ & $\begin{array}{l}0.8498 \\
0.9148 \\
0.9338\end{array}$ & $\begin{array}{l}0.8683 \\
0.9186\end{array}$ & $\begin{array}{l}0.8814 \\
0.9219\end{array}$ & $\begin{array}{l}0.8912 \\
0.9248\end{array}$ & 0.7020 \\
\hline 20 & $\begin{array}{l}0.6393 \\
0.9011 \\
0.9292\end{array}$ & $\begin{array}{l}0.7713 \\
0.9073 \\
0.9314\end{array}$ & $\begin{array}{l}0.8237 \\
0.9124 \\
0.9335\end{array}$ & $\begin{array}{l}0.8523 \\
0.9167 \\
0.9354\end{array}$ & $\begin{array}{l}0.8708 \\
0.9205 \\
0.9371\end{array}$ & $\begin{array}{l}0.8837 \\
0.9237\end{array}$ & $\begin{array}{l}0.8934 \\
0.9266\end{array}$ & 0.7043 \\
\hline 21 & $\begin{array}{l}0.6427 \\
0.9030 \\
0.9307\end{array}$ & $\begin{array}{l}0.7742 \\
0.9091 \\
0.9330\end{array}$ & $\begin{array}{l}0.8263 \\
0.9142 \\
0.9350\end{array}$ & $\begin{array}{l}0.8548 \\
0.9185 \\
0.9369\end{array}$ & $\begin{array}{l}0.8730 \\
0.9222 \\
0.9385\end{array}$ & $\begin{array}{l}0.8857 \\
0.9254 \\
0.9401\end{array}$ & $\begin{array}{l}0.8955 \\
0.9282\end{array}$ & 0.7065 \\
\hline 22 & $\begin{array}{l}0.6459 \\
0.9048 \\
0.9322\end{array}$ & $\begin{array}{l}0.7769 \\
0.9109 \\
0.9344\end{array}$ & $\begin{array}{l}0.8287 \\
0.9159 \\
0.9364\end{array}$ & $\begin{array}{l}0.8570 \\
0.9201 \\
0.9382\end{array}$ & $\begin{array}{l}0.8751 \\
0.9237 \\
0.9399\end{array}$ & $\begin{array}{l}0.8879 \\
0.9269 \\
0.9414\end{array}$ & $\begin{array}{l}0.8974 \\
0.9297 \\
0.9428\end{array}$ & 0.7086 \\
\hline
\end{tabular}


TABLE 1 (contd.)

\begin{tabular}{|c|c|c|c|c|c|c|c|c|}
\hline 1 & & & & 2 & & & & 3 \\
\hline 23 & $\begin{array}{l}0.6490 \\
0.9065 \\
0.9335 \\
0.9453\end{array}$ & $\begin{array}{l}0.7794 \\
0.9125 \\
0.9357\end{array}$ & $\begin{array}{l}0.8310 \\
0.9174 \\
0.9377\end{array}$ & $\begin{array}{l}0.8591 \\
0.9216 \\
0.9395\end{array}$ & $\begin{array}{l}0.8771 \\
0.9252 \\
0.9411\end{array}$ & $\begin{array}{l}0.8897 \\
0.9283 \\
0.9426\end{array}$ & $\begin{array}{l}0.8991 \\
0.9311 \\
0.9440\end{array}$ & 0.7106 \\
\hline 24 & $\begin{array}{l}0.6518 \\
0.9081 \\
0.9348 \\
0.9464\end{array}$ & $\begin{array}{l}0.7817 \\
0.9140 \\
0.9370 \\
0.9476\end{array}$ & $\begin{array}{l}0.8331 \\
0.9189 \\
0.9389\end{array}$ & $\begin{array}{l}0.8610 \\
0.9230 \\
0.9407\end{array}$ & $\begin{array}{l}0.8789 \\
0.9266 \\
0.9423\end{array}$ & $\begin{array}{l}0.8914 \\
0.9297 \\
0.9438\end{array}$ & $\begin{array}{l}0.9008 \\
0.9324 \\
0.9452\end{array}$ & 0.7124 \\
\hline 25 & $\begin{array}{l}0.6545 \\
0.9096 \\
0.9360 \\
0.9475\end{array}$ & $\begin{array}{l}0.7839 \\
0.9154 \\
0.9381 \\
0.9486\end{array}$ & $\begin{array}{l}0.8350 \\
0.9203 \\
0.9401 \\
0.9497\end{array}$ & $\begin{array}{l}0.8629 \\
0.9243 \\
0.9418\end{array}$ & $\begin{array}{l}0.8806 \\
0.9279 \\
0.9434\end{array}$ & $\begin{array}{l}0.8930 \\
0.9309 \\
0.9449\end{array}$ & $\begin{array}{l}0.9023 \\
0.9336 \\
0.9462\end{array}$ & 0.7142 \\
\hline 26 & $\begin{array}{l}0.6570 \\
0.9110 \\
0.9371 \\
0.9482\end{array}$ & $\begin{array}{l}0.7860 \\
0.9167 \\
0.9392 \\
0.9496\end{array}$ & $\begin{array}{l}0.8369 \\
0.9215 \\
0.9411 \\
0.9506\end{array}$ & $\begin{array}{l}0.8646 \\
0.9256 \\
0.9428 \\
0.9516\end{array}$ & $\begin{array}{l}0.8822 \\
0.9291 \\
0.9444\end{array}$ & $\begin{array}{l}0.8946 \\
0.9321 \\
0.9459\end{array}$ & $\begin{array}{l}0.9038 \\
0.9247 \\
0.9472\end{array}$ & 0.7159 \\
\hline 27 & $\begin{array}{l}0.6595 \\
0.9123 \\
0.9381 \\
0.9493\end{array}$ & $\begin{array}{l}0.7879 \\
0.9180 \\
0.9402 \\
0.9505\end{array}$ & $\begin{array}{l}0.8386 \\
0.9227 \\
0.9421 \\
0.9515\end{array}$ & $\begin{array}{l}0.8662 \\
0.9268 \\
0.9438 \\
0.9525\end{array}$ & $\begin{array}{l}0.8837 \\
0.9302 \\
0.9454 \\
0.9534\end{array}$ & $\begin{array}{l}0.8960 \\
0.9332 \\
0.9468\end{array}$ & $\begin{array}{l}0.9051 \\
0.9358 \\
0.9481\end{array}$ & 0.7174 \\
\hline 28 & $\begin{array}{l}0.6617 \\
0.9135 \\
0.9391 \\
0.9502\end{array}$ & $\begin{array}{l}0.7898 \\
0.9192 \\
0.9412 \\
0.9513\end{array}$ & $\begin{array}{l}0.8402 \\
0.9239 \\
0.9431 \\
0.9524\end{array}$ & $\begin{array}{l}0.8677 \\
0.9279 \\
0.9447 \\
0.9533\end{array}$ & $\begin{array}{l}0.8851 \\
0.9313 \\
0.9463 \\
0.9542\end{array}$ & $\begin{array}{l}0.8973 \\
0.9342 \\
0.9477 \\
0.9550\end{array}$ & $\begin{array}{l}0.9064 \\
0.9368 \\
0.9490\end{array}$ & 0.7190 \\
\hline 29 & $\begin{array}{l}0.6639 \\
0.9146 \\
0.9401 \\
0.9510\end{array}$ & $\begin{array}{l}0.7915 \\
0.9202 \\
0.9421 \\
0.9521\end{array}$ & $\begin{array}{l}0.8418 \\
0.9250 \\
0.9439 \\
0.9531\end{array}$ & $\begin{array}{l}0.8691 \\
0.9289 \\
0.9456 \\
0.9540\end{array}$ & $\begin{array}{l}0.8865 \\
0.9323 \\
0.9471 \\
0.9550\end{array}$ & $\begin{array}{l}0.8986 \\
0.9352 \\
0.9485 \\
0.9558\end{array}$ & $\begin{array}{l}0.9076 \\
0.9378 \\
0.9498 \\
0.9566\end{array}$ & 0.7204 \\
\hline 30 & $\begin{array}{l}0.6660 \\
0.9157 \\
0.9409 \\
0.9518 \\
0.9581\end{array}$ & $\begin{array}{l}0.7932 \\
0.9213 \\
0.9430 \\
0.9529\end{array}$ & $\begin{array}{l}0.8432 \\
0.9260 \\
0.9448 \\
0.9539\end{array}$ & $\begin{array}{l}0.8734 \\
0.9299 \\
0.9464 \\
0.9548\end{array}$ & $\begin{array}{l}0.8877 \\
0.9332 \\
0.9479 \\
0.9557\end{array}$ & $\begin{array}{l}0.8998 \\
0.9361 \\
0.9493 \\
0.9566\end{array}$ & $\begin{array}{l}0.9088 \\
0.9387 \\
0.9506 \\
0.9573\end{array}$ & 0.7218 \\
\hline
\end{tabular}

The solution of problem (b) can be formulated as the following

THEOREM 2. There exists a sequence of numbers $\left\{\alpha_{n}\right\}_{n=1}^{\infty}$ such that

(i) $\tau^{*}=\inf \left\{n: \xi_{n} \geqslant a_{n}\right\}$ is the optimal stopping time, i.e., (5) holds;

(ii) $\lim _{n \rightarrow \infty} a_{n}=A, a_{n} \leqslant a_{n+1}$ for each $n \in N$;

(iii) the sequence $\left\{a_{n}\right\}_{n=1}^{\infty}$ satisfies the recurrence relation

$$
a_{n}^{n}=\frac{n+1}{n+2} \alpha_{n+1}^{n+2}+\frac{A^{n+2}}{n+2}
$$


(iv) $a_{n}$ can be obtained as the limit of the sequence of unique solutions. $\beta_{n}^{k} \in\left(0, \beta_{n+1}^{k-1}\right)$ of the equation

$$
\begin{aligned}
& \frac{n+k}{n+2 k}\left[x^{n+2 k}-\left(\beta_{n+1}^{k-1}\right)^{n+2 k}\right]+\frac{n+1}{n+2}\left(\beta_{n+1}^{k-1}\right)^{n+2}+\frac{A^{n+2}}{n+2}-x^{n}=0 \\
& \text { as } k \rightarrow \infty ;
\end{aligned}
$$

(v) $v=v(0,0)=P\left\{A \geqslant \xi_{\tau^{*}}>\eta_{\tau^{*}}\right\}=\frac{1}{2}\left(\alpha_{1}^{2}+A^{2}\right)$.

Proof. By Lemma 2 we have

$$
v(n, x)=\lim _{N \rightarrow \infty} Q^{N} g(n, x) .
$$

For the investigated problem, $Q g(n, x)$ takes the form

$$
\begin{aligned}
Q g(n, x)=\max \{g(n, x), & T g(n, x)\} \\
= & \begin{cases}\frac{n+1}{n+2} x^{n+2}+\frac{A^{n+2}}{n+2} & \text { for } x<\beta_{n}^{1}, \\
x^{n} & \text { for } \beta_{n}^{1} \leqslant x \leqslant A, \\
0 & \text { for } x>A,\end{cases}
\end{aligned}
$$

where $\beta_{n}^{1}$ is the unique solution of the equation

$$
\frac{n+1}{n+2} x^{n+2}+\frac{A^{n+2}}{n+2}-x^{n}=0 \quad \text { in }(0, A) \text {. }
$$

Equation (6) has a unique solution in $(0, A)$ because the function

$$
\varphi_{1}(n, x)=\frac{n+1}{n+2} x^{n+2}+\frac{A^{n+2}}{n+2}-x^{n}
$$

has the following properties:

(a) $\varphi_{1}(n, 0)>0, \varphi_{1}(n, A)=A^{n}(1-A)<0$;

(b) $\varphi_{1}(n, x)$ has in $(0,1)$ a unique minimum at $x=\sqrt{n /(n+1)}$. Since

$$
\begin{aligned}
\varphi_{1}(n+1, x) & =\frac{n+2}{n+3} x^{n+3}+\frac{A^{n+3}}{n+3}-x^{n+1} \\
& =x \varphi_{1}(n, x)+\frac{1}{(n+3)(n+2)} x^{n+3}-\frac{A^{n+2}}{n+2} x+\frac{A^{n+3}}{n+3} \\
& \stackrel{\mathrm{d} f}{=} x \varphi_{1}(n, x)+q(n, x)
\end{aligned}
$$

and $q(n, x)>0$ for $x \in(0, A)$, we have $\varphi_{1}\left(n+1, \beta_{n}^{1}\right)>0$, and hence $\beta_{n}^{1}<\beta_{n+1}^{1}$. 
We calculate $Q^{N} g(n, x)$ by induction:

$$
\begin{aligned}
Q^{N} g(n, x) & =\max \left\{Q^{N-1} g(n, x), T Q^{N-1} g(n, x)\right\} \\
& =\max \left\{g(n, x), T Q^{N-1} g(n, x)\right\} .
\end{aligned}
$$

Let us put

$$
\varphi_{N}(n, x)=T Q^{N-1} g(n, x)-g(n, x) .
$$

Assume by induction that there exist unique solutions $\beta_{n}^{r}$ of the equations $\varphi_{r}(n, x)=0$ in $(0, A)$ for $r=1,2, \ldots, k, \beta_{n}^{1}<\beta_{n}^{2}<\ldots<\beta_{n}^{k}$, and $\beta_{n}^{r}<\beta_{n+1}^{r}$ for $r=1,2, \ldots, k$ and $n=1,2, \ldots$ We have then

$$
= \begin{cases}\frac{n+k}{n+2 k}\left[x^{n+2 k}-\left(\beta_{n+1}^{k-1}\right)^{n+2 k}\right]+\frac{n+1}{n+2}\left(\beta_{n+1}^{k-1}\right)^{n+2}+\frac{A^{n+2}}{n+2} & \text { for } x<\beta_{n}^{k}, \\ x^{n} & \text { for } \beta_{n}^{k} \leqslant x \leqslant A, \\ 0 & \text { for } x>A .\end{cases}
$$

For $x<\beta_{n+1}^{k}$ we obtain

$$
\begin{aligned}
T Q^{k} g(n, x)=x & \frac{n+k+1}{n+2 k+1}\left[x^{n+2 k+1}-\left(\beta_{n+2}^{k-1}\right)^{n+2 k+1}\right]+\frac{n+2}{n+3}\left(\beta_{n+2}^{k-1}\right)^{n+3}+ \\
& \left.+\frac{A^{n+3}}{n+3}\right\}+\int_{x}^{\beta_{n+1}^{k}}\left\{\frac{n+k+1}{n+2 k+1}\left[y^{n+2 k+1}-\left(\beta_{n+2}^{k-1}\right)^{n+2 k+1}\right]+\right. \\
& \left.+\frac{n+2}{n+3}\left(\beta_{n+2}^{k-1}\right)^{n+3}+\frac{A^{n+3}}{n+3}\right\} d y+\int_{\beta_{n+1}^{k}}^{A} y^{n+1} d y \\
= & \frac{n+k+1}{n+2(k+1)}\left[x^{n+2(k+1)}-\left(\beta_{n+1}^{k}\right)^{n+2(k+1)}\right]+\frac{n+1}{n+2}\left(\beta_{n+1}^{k}\right)^{n+2}+ \\
& +\frac{A^{n+2}}{n+2}+\left(\beta_{n+1}^{k}\right)\left\{\frac{n+k+1}{n+2 k+1}\left[\left(\beta_{n+1}^{k}\right)^{n+2 k+1}-\left(\beta_{n+2}^{k-1}\right)^{n+2 k+1}\right]+\right. \\
& \left.+\frac{n+2}{n+3}\left(\beta_{n+2}^{k-1}\right)^{n+3}+\frac{A^{n+3}}{n+3}-\left(\beta_{n+1}^{k}\right)^{n+1}\right\} \\
= & \frac{n+k+1}{n+2(k+1)}\left[x^{n+2(k+1)}-\left(\beta_{n+1}^{k}\right)^{n+2(k+1)}\right]+\frac{n+1}{n+2}\left(\beta_{n+1}^{k}\right)^{n+2}+ \\
& +\frac{A^{n+2}}{n+2}+\left(\beta_{n+1}^{k}\right) \varphi_{k}\left(n+1, \beta_{n+1}^{k}\right) \\
= & \frac{n+k+1}{n+2(k+1)}\left[x^{n+2(k+1)}-\left(\beta_{n+1}^{k}\right)^{n+2(k+1)}\right]+ \\
& +\frac{n+1}{n+2}\left(\beta_{n+1}^{k}\right)^{n+2}+\frac{A^{n+2}}{n+2} .
\end{aligned}
$$


Therefore, for $x<\beta_{n+1}^{k}$ the function $\varphi_{k+1}(n, x)$ is given by the formula

$$
\begin{aligned}
\varphi_{k+1}(n, x)= & \frac{n+k+1}{n+2(k+1)}\left[x^{n+2(k+1)}-\left(\beta_{n+1}^{k}\right)^{n+2(k+1)}\right]+ \\
& +\frac{n+1}{n+2}\left(\beta_{n+1}^{k}\right)^{n+2}+\frac{A^{n+2}}{n+2}-x^{n}
\end{aligned}
$$

The function $\varphi_{k+1}(n, x)$ has the following properties:

(a) $\varphi_{k+1}(n, x)=T Q^{k} g(n, x)-g(n, x)=T g(n, x)-g(n, x)=\varphi_{k}(n, x)$ $<0$ for $x \geqslant \beta_{n+1}^{k}>\beta_{n}^{k}$.

(b) $\varphi_{k+1}(n, x)>\varphi_{k}(n, x) \geqslant 0$ for $x \leqslant \beta_{n}^{k}$.

For $x<\beta_{n}^{k}, Q^{k} g(n, x)>g(n, x)$ and $Q^{k+1} g(n, x) \geqslant Q^{k} g(n, x)$. Then, for $x<\beta_{n}^{k}, Q^{k+1} g(n, x)>g(n, x)$. Consequently, we have (b).

(c) The polynomial defined by (8) has a unique minimum in $(0,1)$. . From (a)-(c) we infer that the equation $\varphi_{k+1}(n, x)=0$ has the unique root $\beta_{n}^{k+1}$ and

$$
\beta_{n}^{k}<\beta_{n}^{k+1}<\beta_{n+1}^{k} \quad \text { for } n=1,2, \ldots
$$

Formula (9) implies $\beta_{n+1}^{k}<\beta_{n+1}^{k+1}<\beta_{n+2}^{k}$. Therefore $\beta_{n}^{k+1}<\beta_{n+1}^{k+1}$. Moreover,

$$
\begin{aligned}
& Q^{k+1} g(n, x) \\
& =\left\{\begin{array}{lr}
\frac{n+k+1}{n+2(k+1)}\left[x^{n+2(k+1)}-\left(\beta_{n+1}^{k}\right)^{n+2(k+1)}\right]+\frac{n+1}{n+2}\left(\beta_{n+1}^{k}\right)^{n+2}+\frac{A^{n+2}}{n+2} \\
x^{n} & \text { for } x<\beta_{n}^{k+1}, \\
0 & \text { for } \beta_{n}^{k+1} \leqslant x \leqslant A, \\
& \text { for } x>A .
\end{array}\right.
\end{aligned}
$$

The sequence $\left(\beta_{n}^{k}\right)_{k=1}^{\infty}$ is monotone and bounded by $A$, so the limit

$$
\lim _{k \rightarrow \infty} \beta_{n}^{k}=a_{n}
$$

exists. Hence

$$
v(n, x)=\lim _{k \rightarrow \infty} Q^{k} g(n, x)= \begin{cases}\frac{n+1}{n+2} a_{n+1}^{n+2}+\frac{A^{n+2}}{n+2} & \text { for } x<a_{n} \\ x^{n} & \text { for } a_{n} \leqslant x \leqslant A \\ 0 & \text { for } x>A\end{cases}
$$


We prove now that the function $v(n, x)$ is continuous at $x=a_{n}$. To the contrary, let

$$
a_{n}^{n}>\frac{n+1}{n+2} a_{n+1}^{n+2}+\frac{A^{n+2}}{n+2} .
$$

Then there exists $\alpha_{n}^{\prime}<\alpha_{n}$ such that

$$
v_{1}(n, x)= \begin{cases}\frac{n+1}{n+2} a_{n+1}^{n+2}+\frac{A^{n+2}}{n+2} & \text { for } x<a_{n}^{\prime}, \\ x^{n} & \text { for } \alpha_{n}^{\prime} \leqslant x \leqslant A, \\ 0 & \text { for } x>A\end{cases}
$$

and

$$
v_{1}(n, x)>v(n, x)=\sup _{\tau \in \mathbb{P}_{n}} \mathrm{E}_{x} g\left(\tau, \xi_{\tau}\right) \quad \text { for } x \in\left(a_{n}^{\prime}, \alpha_{n}\right)
$$

in spite of the definition of $v(n, x)$.

The proof of the converse inequality in (10) is similar. We have then

$$
a_{n}^{n}=\frac{n+1}{n+2} a_{n+1}^{n+2}+\frac{A^{n+2}}{n+2} \quad \text { and } \quad \frac{A \sqrt[n]{A^{2}}}{\sqrt[n]{n+2}}<a_{n}<A \sqrt[n]{A^{2}} .
$$

Consequently,

$$
\lim _{n \rightarrow \infty} \alpha_{n}=A \text {. }
$$

From Lemma 2 we infer that $\tau_{0}^{*}=\inf \left\{n: \xi_{n} \geqslant \alpha_{n}\right\}$ is the optimal stopping time only if $P\left\{\tau_{0}^{*}<\infty\right\}=1$.

We have

$$
P\left\{\tau_{0}^{*}<\infty\right\}=P\left\{\bigcup_{n=1}^{\infty}\left\{\xi_{n} \geqslant a_{n}\right\}\right\} \geqslant P\left\{\bigcup_{n=1}^{\infty}\left\{\xi_{n} \geqslant A\right\}\right\}=\lim _{n \rightarrow \infty} P\left\{\xi_{n} \geqslant A\right\}=1 .
$$

Assume that $P\left\{\xi_{0}=0\right\}=1$. Then

$$
\begin{aligned}
v & =v(0,0)=\mathrm{E}_{0} v\left(1, \xi_{1}\right)=P\left\{A \geqslant \xi_{\tau^{*}}>\eta_{\tau^{*}}\right\}=\int_{0}^{a_{1}}\left(\frac{2}{3} a_{2}^{3}+\frac{A^{3}}{3}\right) d y+\int_{a_{1}}^{A} y d y \\
& =a_{1}\left(\frac{2}{3} a_{2}^{3}+\frac{A^{3}}{3}-a_{1}\right)+\frac{1}{2}\left(a_{1}^{2}+A^{2}\right)=\frac{1}{2}\left(a_{1}^{2}+A^{2}\right) .
\end{aligned}
$$

This completes the proof of Theorem 2.

Let $0<A<1$. We calculate $\tau^{*} \in \mathfrak{M}$ such that

$$
P\left\{\min \left(A, \xi_{\tau^{*}}\right)>\eta_{\tau^{*}}\right\}=\sup _{\tau \in \mathbb{M}} P\left\{\min \left(A, \xi_{\tau}\right)>\eta_{\boldsymbol{\tau}}\right\}
$$


Let us put

$$
Z_{n}=P\left\{\min \left(A, \xi_{n}\right)>\eta_{n} \mid \mathscr{F}_{n}\right\}=g\left(n, \xi_{n}\right)= \begin{cases}A^{n} & \text { for } \xi_{n}>A \\ \xi_{n}^{n} & \text { for } \xi_{n} \leqslant A\end{cases}
$$

Problem (c) has the following solution:

THEOREM 3. There exists a sequence of numbers $\left\{\gamma_{n}\right\}_{n=1}^{\infty}$ such that

(i) $\tau^{*}=\inf \left\{n: \xi_{n} \geqslant \gamma_{n}\right\}$ is the optimal stopping time in (11);

(ii) $\lim _{n \rightarrow \infty} \gamma_{n}=A$ and $\gamma_{n} \leqslant \gamma_{n+1}$ for each $n \in N$;

(iii) the sequence $\left\{\gamma_{n}\right\}_{n=1}^{\infty}$ satisfies the recurrence relation

$$
\gamma_{n}^{n}=\frac{n+1}{n+2}\left(\gamma_{n+1}^{n+2}-A^{n+2}\right)+A^{n+1}
$$

(iv) $\gamma_{n}$ can be obtained as the limit of the sequence of unique solutions $\delta_{n}^{k} \in\left(0, \delta_{n+1}^{k-1}\right)$ of the equation

$$
\begin{aligned}
\frac{n+k}{n+2 k}\left[x^{n+2 k}-\left(\delta_{n+1}^{k-1}\right)^{n+2 k}\right]+\frac{n+1}{n+2}\left[\left(\delta_{n+1}^{k-1}\right)^{n+2}-A^{n+2}\right]+A^{n+1}-x^{n} & =0 \\
\text { as } k & \rightarrow \infty ;
\end{aligned}
$$

(v) $v=v(0,0)=P\left\{\min \left(A, \xi_{\tau^{*}}\right)>\eta_{\tau^{*}}\right\}=\frac{1}{2}\left(\gamma_{1}^{2}+A^{2}\right)+A(1-A)$.

The proof of this theorem is very similar to the proof of Theorem 2 . The details can be found in [4].

3. The optimal stopping of the infinite sequence of maxima with a discount factor. Let $0<a<1$. We want to determine $\tau^{*} \in \mathfrak{W}$ such that

$$
\mathrm{E} \alpha^{\tau^{*}} \chi_{\left\{\xi_{\tau *}>\eta_{\tau *}\right\}}=\sup _{\tau \in \mathfrak{M}} \mathrm{E} \alpha^{\tau} \chi_{\left\{\xi_{\tau}>\eta_{\tau}\right\}}
$$

We write

$$
Z_{n}=\alpha^{n} P\left\{\xi_{n}>\eta_{n} \mid \mathscr{F}_{n}\right\}=g\left(n, \xi_{n}\right)=\alpha^{n} \xi_{n}^{n} .
$$

The solution of problem (d) can be formulated as the following THEOREM 4. There exists a sequence of numbers $\left\{\lambda_{n}\right\}_{n=1}^{\infty}$ such that

(i) $\tau^{*}=\inf \left\{n: \xi_{n} \geqslant \lambda_{n}\right\}$ is the optimal stopping time, i.e., it satisfies (12) $\}$

(ii) the sequence $\left\{\lambda_{n}\right\}_{n=1}^{\infty}$ fulfils the recurrence relation

$$
\lambda_{n}^{n}=a\left(\frac{n+1}{n+2} \lambda_{n+1}^{n+2}+\frac{1}{n+2}\right)
$$

(iii) $\lim _{n \rightarrow \infty} \lambda_{n}=1, \lim _{n \rightarrow \infty}\left(\lambda_{n}\right)^{n}=0$, and $\lambda_{n}^{\top} \leqslant \lambda_{n+1}$ for each $n \in N$; 
(iv) $\lambda_{n}$ can be obtained as the limit of unique solutions $\mu_{n}^{k} \in\left(0, \mu_{n+1}^{k-1}\right)$ of the equation

$$
\begin{aligned}
& \frac{n+k}{n+2 k} \alpha^{n+k}\left[x^{n+2 k}-\left(\mu_{n+1}^{k-1}\right)^{n+2 k}\right]+\frac{n+1}{n+2} \alpha^{n+1}\left(\mu_{n+1}^{k-1}\right)^{n+2}+\frac{a^{n+1}}{n+2}-x^{n}=0 \\
& \text { (v) } v=v(0,0)=E a^{\tau^{*}} \chi_{\left\{\xi_{\tau^{*}}>\eta_{\tau_{*}}\right\}}=\alpha\left(1+\lambda_{1}^{2}\right) / 2 \text {. } \\
& \text { as } k \rightarrow \infty \text {; }
\end{aligned}
$$

Proof. By Lemma 2 we have

$$
v(n, x)=\lim _{N \rightarrow \infty} Q^{N} g(n, x) .
$$

It can be proved, similarly as in the proof of Theorem 2, that there exist unique solutions $\mu_{n}^{r}$ of the equations $\varphi_{r}(n, x)=0$ in $(0,1)$ for $r=1,2, \ldots, k, \mu_{n}^{1}<\mu_{n}^{2}<\ldots<\mu_{n}^{r}, \mu_{n}^{r}<\mu_{n+1}^{r}$ for $r=1,2, \ldots, k$ and $n=1,2, \ldots$, and

$$
Q^{k} g(n, x)=\left\{\begin{array}{cc}
\frac{n+k}{n+2 k} a^{n+k}\left[x^{n+2 k}-\left(\mu_{n+1}^{k-1}\right)^{n+2 k}\right]+\frac{n+1}{n+2} a^{n+1}\left(\mu_{n+1}^{k-1}\right)^{n+2}+ \\
+\frac{a^{n+1}}{n+2} & \text { for } x<\mu_{n}^{k} \\
(\alpha x)^{n} & \text { for } x \geqslant \mu_{n}^{k} .
\end{array}\right.
$$

The sequence $\left\{\mu_{n}^{k}\right\}_{k=1}^{\infty}$ is monotone and bounded by 1 , so the limit

$$
\lim _{k \rightarrow \infty} \mu_{n}^{k}=\lambda_{n}
$$

exists. Therefore, we have

$$
v(n, x)=\lim _{k \rightarrow \infty} Q^{k} g(n, x)= \begin{cases}\frac{n+1}{n+2} a^{n+1} \lambda_{n+1}^{n+2}+\frac{a^{n+1}}{n+2} & \text { for } x<\lambda_{n} \\ (\alpha x)^{n} & \text { for } x \geqslant \lambda_{n}\end{cases}
$$

We can see, as in the proof of Theorem 2, that the function $v(n, x)$ is continuous at $x=\lambda_{n}$, i.e., $\lambda_{n}$ fulfils the following recurrence relation:

$$
\left(\alpha \lambda_{n}\right)^{n}=\frac{n+1}{n+2} \alpha^{n+1} \lambda_{n+1}^{n+2}+\frac{\alpha}{n+2}, \quad n=1,2, \ldots
$$

Hence

$$
\frac{\sqrt[n]{\alpha}}{\sqrt[n]{n+2}}<\lambda_{n}<\sqrt[n]{\alpha} \quad \text { and } \quad \lim _{n \rightarrow \infty} \lambda_{n}=1
$$


From (13) we infer by induction that for each $k \in N$

$$
\lambda_{n}^{n} \leqslant \alpha^{k}+\frac{1}{n+2} \frac{a\left(1-a^{k-1}\right)}{1-\alpha}
$$

Hence

$$
\lambda_{n}^{n} \leqslant \alpha^{k}+\frac{1}{n+2} \frac{\alpha}{1-\alpha}
$$

and

$$
\lambda_{n}^{n} \leqslant \inf _{k}\left\{a^{k}+\frac{1}{n+2} \frac{a}{1-a}\right\}=\frac{1}{n+2} \frac{a}{1-a} .
$$

Then

$$
\lim _{n \rightarrow \infty} \lambda_{n}^{n}=0 \text {. }
$$

Now we can show that $\tau^{*}=\inf \left\{n: \xi_{n} \geqslant \lambda_{n}\right\}$ is finite with probability 1 . We have

$$
\begin{aligned}
P\left\{\tau^{*}<\infty\right\} & =P\left\{\bigcup_{n=1}^{\infty}\left\{\xi_{n} \geqslant \lambda_{n}\right\}\right\} \geqslant \lim _{N \rightarrow \infty} P\left\{\xi_{N}^{N} \geqslant \lambda_{N}^{N}\right\} \\
& =\lim _{N \rightarrow \infty} P\left\{\xi_{N}^{N} \geqslant \lambda_{N}^{N}\right\}=\lim _{N \rightarrow \infty}\left(1-\lambda_{N}^{N}\right)=1
\end{aligned}
$$

because $\xi_{n}^{n}$ is uniformly distributed on $[0,1]$.

Assume that $P\left\{\xi_{0}=0\right\}=1$. Then

$$
\begin{aligned}
v & =v(0,0)=\mathrm{E}_{0} v\left(1, \xi_{1}\right)=\int_{0}^{\lambda_{1}}\left(\frac{2}{3} \alpha^{2} \lambda_{2}^{3}+\frac{\alpha^{2}}{3}\right) d y+\int_{\lambda_{1}}^{1} \alpha y d y \\
& =\left(\frac{2}{3} \alpha^{2} \lambda_{2}^{3}+\frac{\alpha^{2}}{3}\right) \lambda_{1}+\alpha \frac{1-\lambda_{1}^{2}}{2}-\lambda_{1}\left(\frac{2}{3} \alpha^{2} \lambda_{2}^{3}+\frac{\alpha^{2}}{3}-\alpha \lambda_{1}\right)=\frac{\alpha}{2}\left(1-\lambda_{1}^{2}\right) .
\end{aligned}
$$

This completes the proof of Theorem 4 .

Acknowledgment. The author would like to express his sincere thanks to Professor S. Trybuła for suggesting the problem. The author is also indebted to Dr. M. Kurzyński for his help in computing the table.

\section{References}

[1] T. Bojdecki, A note on the optimal stopping of sums of independent random variables, Bull. Acad. Polon. Sci., Sér. sci. math., astronom., phys., 25 (1977), p. 833-837.

[2] - On optimal stopping of a sequence of independent random variables - probability maximizing approach, Stochastic Processes Appl. 6 (1978), p. 153-163. 
[3] A. N. Širjaev (А. Н. Ш ир я ев), Статистический последоватељънй анализ, „Наука", Москва 1969.

[4] K. Szajowski, Wybrane problemy optymalnego zatrzymywania ciagu zmiennych losowych (Thesis), Rap. Ser. PRE nr 32, Institute of Mathematics, Technical University of Wrocław, 1979.

INSTITUTE OF MATHEMATICS

THOHNICAL UNIVERSITY OF WROCEAW

50-370 WROCEAW

Received on 15. 5. 1981 